
\title{
25 Research Square \\ Effect and Mechanism of PLSCR4 in The Injury of Human Pulmonary Microvascular Endothelial Cells Induced by LPS
}

\section{Xiaobin Liu}

Shandong University https://orcid.org/0000-0002-1626-2414

\section{Dong Wang}

Shandong Qianfoshan Hospital

\section{Xiaoning Zhang}

Shandong University

\section{Changping Gu}

Shandong Qianfoshan Hospital

\section{Fan Yang}

Shandong Qianfoshan Hospital

Yuelan Wang ( $\square$ wyl0910@126.com )

Shandong Qianfoshan Hospital

\section{Research}

Keywords: Lipopolysaccharide, PLSCR4, Human pulmonary microvascular endothelial cell, pyroptosis, GSDMD

Posted Date: October 27th, 2020

DOI: https://doi.org/10.21203/rs.3.rs-96163/v1

License: (c) (i) This work is licensed under a Creative Commons Attribution 4.0 International License. Read Full License 


\section{Abstract}

Background: Previous experiments have revealed that Phospholipid scramblase 4 (PLSCR4) mRNA significantly increased in ARDS model of human pulmonary microvascular endothelial cells (HPMECs) stimulated by lipopolysaccharide (LPS), but the specific effect and mechanism have not been reported to date.In consideration of the function of PLSCR4 that has been reported, we infer that PLSCR4 may have impact on cell death through pyroptosis.

Methods: To investigate the effect and mechanism of PLSCR4 in ARDS in vitro, HPMECs were cultured in 6well plates and divded into four groups: control group, LPS group ,siRNA+LPS group and transfection reagent group. After $4 \mathrm{~h}$ of LPS stimulation, western blot was used to detect the expression of PLSCR4 and pyroptosis - relevant proteins; Using ELISA to exame IL-1 $\beta$ and IL-18; Tracer flux assays was used to evaluate the barrier function of endothelium, and fluorescence assays to observe the distribution of phospholipid and the degree of pyroptosis. At last we used DNA pull-down and protein profiling to study the potential transcription factor of PLSCR4.

Results: It was found that when the expression of PLSCR4 was elevated, the concentration of IL-1 $\beta$ and IL-18 decreased, together with barrier damage ( $P<0.05$ ), and HPMECs injury was reduced with more distribution of phospholipid on the external sie of cells, but the pyroptosis-relevant proteins did not change obviously ( $P$ $<0.05$ ); then we found P62280 remarkably increased in the DNA pull-down assay, and it could impact the pyroptosis by regulating the expression of PLSCR4.

Conclusion: We found that PLSCR4 alleviated pyroptosis by transporting phosphatidylserine (PS) to the outside of the membrane, blocking the forming of pyroptosis pores composed of gasdermin $D$, it was further found that the expression level of PLSCR4 during LPS stimulation was regulated by P62280. This study for the first time explains the protective effect of PLSCR4 in the ARDS model, and its possible mechanism, providing a new research idea for the clinical treatment of ARDS.

\section{Introduction}

Acute respiratory distress syndrome (ARDS) with inflammatory injury as the main feature is one of the main causes of death in patients with multiple organ dysfunction caused by sepsis, which has high morbidity and mortality $(1,2)$.The main pathological characteristics were the release of inflammatory factors, the infiltration and aggregation of inflammatory cells and the damage of the vascular endothelium that ultimately lead to changes in pulmonary microvascular endothelial permeability and pulmonary edema(3).

Despite the extensive researches on ARDS, presently there are no effective drug treatments(1). In the prior study of our group, high-throughput cell sequencing and bioinformatics analysis were carried out, and the results revealed that the mRNA expression of PLSCR4 protein significantly increased, and it was at the central position $(4,5)$ in the co-expression network diagram, suggesting that PLSCR4 may play an important role in the pathological process of the pulmonary microvascular endothelium induced by LPS (Fig. 1). However, the specific role and mechanism have not been reported, to date. 
PLSCRs are a group of single transmembrane proteins that could mediate nonspecific, bidirectional, and headgroup independent transbilayer movement of phospholipid( PS ) across the lipid bilayers among which PS could be transported across the membrane in a $\mathrm{Ca}^{2+}$-dependent manner, increasing its exposures $(6,7)$. The exofacical exposure of PS has been considered to be an important marker, and plays an important part in the pathological process of multiple cell types(8). In recent years, some literatures have reported that in the process of inflammatory cell death-pyroptosis, the function of pyroptosis executive protein Gasdermin D (GSDMD) may have a significant relationship with PS(9). Therefore, this study aimed to explore the biological effect and mechanism of PLSCR4 in the process of acute pulmonary microvascular endothelial injury caused by LPS, and its relationship with pyroptosis.

\section{Materials And Methods}

\section{Kits, antibodies and reagents}

PLSCR4 siRNA was synthesized and supplied by GenePharma. The PLSCR4 antibody was purchased from Abcam. GSDMD, caspase-1 and caspase-1 P20 antibodies were purchased from Cell Signaling Technology. The IL-1 $\beta$ and IL-18 ELISA detection kits were provided by Elabscience Biotechnology Co., Ltd., Wuhan. The annexin V-FITC cell pyroptosis detection kit was purchased from Beyotime Biotechnology. The transwell filter insert for tracer flux assays was purchased from Corning (Corning 3401, 12-mm diameter, 0.4- $\mathrm{mm}$ pore size). The fluorescein isothiocyanate (FITC)-dextran was purchased from Sigma-Aldrich.

\section{Cell culture}

The HPMECs were purchased from ScienCell Research Laboratories. The cell recovery, culture, and subcultre were carried out in at $37^{\circ} \mathrm{C}$ with $5 \% \mathrm{CO}_{2}$ in a cell incubator, according to the standard operating instructions provided by ScienCell Research Laboratories. In our study, the cell culture medium, serum, growth factor and antibiotics were all recommended by ScienCell. When the culture reached $80 \%-90 \%$ confluency, LPS was added to the working solution in the wells of experimental groups to reach a final concentration of $1 \mu \mathrm{g} / \mathrm{ml}$, and cultured for four hours.

\section{Small interfering RNA (siRNA)-mediated knockdown}

Cells were seeded in a six-well plate at the day before the transfection, and the cell growth was observed next day. When the confluency reached $50 \%-70 \%$, the HPMEC can be transfected. A nuclease-free EP tube was prepared in advance. Then, $200 \mu$ of the basic medium was added to the EP tube, and $5.5 \mu$ of $20 \mu \mathrm{M}$ of siRNA was added. After mix by pipetting up and down, $16 \mu \mathrm{l}$ of transfection reagent INTERFERin (polyplustransfection) was added and incubated at room temperature for 10 minutes. Then, the culture medium was changed during incubation, the six-well plate was rinsed with sterile DPBS solution twice, and $2 \mathrm{ml}$ of fresh complete medium was added. Afterwards, the transfection complex was added to the six-well plate after incubation. The concentration of the siRNA was $50 \mathrm{nM}$. After mixing, these cells were incubated at $37^{\circ} \mathrm{C}$ for two days, then detect the protein expression.

\section{Western blot}


$100 \mu$ of cell lysate (RIPA:PMSF=100:1) was added in each well of the six-well plate, and set the plate on ice for 30 minutes. After the lysis, the cell lysate was collected in a 1.5-ml EP tube. Subseqeuntly, the centrifuge processing of the lysate was carried out at $12,000 \mathrm{rpm}$ for 15 minutes at $4^{\circ} \mathrm{C}$. After centrifugation, the supernatant was transferred to another EP tube to obtain the total protein. The total protein concentration was detected using the BCA Protein Assay Kit, and $5 \times$ loading buffer was added to the protein samples, which was then heated and denatured at $96^{\circ} \mathrm{C}$ for five minutes. The obtained protein samples were subjected to SDS-PAGE gel electrophoresis, and transferred into PVDF membranes. Then the membranes were blocked with $5 \%$ skimmed milk for one hour at room temperature, and the sample was washed with TBST for five minutes for three times at the end of blocking. Incubate the membranes in the primary antibody diluent $(1: 1,000)$ overnight at $4^{\circ} \mathrm{C}$. On the next day, incubate the membranes in the secondary antibody diluent $(1: 5,000)$ at room temperature for one hour, and finally set on the scan machine(10).

\section{ELISA detection of IL-1 $\beta$ and IL-18}

After four hours of stimulation by LPS, the supernatant of each group of cells in the six-well plate was collected and added to a EP tube. The supernatant was centrifuged at 1,000 g for 20 minutes at $4^{\circ} \mathrm{C}$ to remove the impurities and cell debris. The double-antibody sandwich ELISA kit was used for the detection of inflammatory factors IL-1 $\beta$ and IL-18(11), which were used to assess the pyroptosis. If these could not be detected in time, the sample was frozen at $-80^{\circ} \mathrm{C}$ to avoid repeated freezing and thawing.

\section{Tracer flux assays}

In this study, Tracer Flux Assays were performed to detect the changes in endothelial permeability. The Transwell chamber was purchased from Corning, USA, with a filter pore diameter of $0.4 \mu \mathrm{m}$ and a cell diameter of $12 \mathrm{~mm}$. Next, $1.5 \mathrm{ml}$ of complete medium was added at the bottom chamber, and $0.5 \mathrm{ml}$ of complete culture medium that contained the suspended cells were added to the upper chamber. The number of pulmonary microvascular endothelial cells was $5 \times 10^{5}$. Afterwards, the Transwell chamber was cultured in an incubator at $37^{\circ} \mathrm{C}$. On the next day, the medium was changed, and the cell adhesion was observed under a microscope. After 1-3 days, when the cell confluency reached $90 \%$, the medium in the bottom layer for the experimental group was replaced with fresh medium containing $1 \mu \mathrm{g} / \mathrm{ml}$ of LPS, while the medium in the upper chamber was replaced with fresh culture containing $1 \mu \mathrm{g} / \mathrm{ml}$ of LPS and $1 \mathrm{mg} / \mathrm{ml}$ of FITC-dextran. The upper and lower layers of the chamber for the blank control group did not contain LPS, only $1 \mathrm{mg} / \mathrm{ml} \mathrm{of} \mathrm{FITC-}$ dextran in the upper layer. Both groups were placed in a cell incubator at $37^{\circ} \mathrm{C}$ to continue culturing for four hours. After four hours, $50 \mu \mathrm{l}$ of the bottom culture medium of each group was taken and added to a 96-well plate. After labeling, the samples were placed in a fluorescence microplate reader (BioTek SynergeH1M, USA) to read the data. The excitation light wavelength of the microplate reader was set to $488 \mathrm{~nm}$, and the emission wavelength was set to $520 \mathrm{~nm}(12)$. Data analysis was expressed by permeability index $\mathbb{P} \mathrm{PI}$, that is, the ratio of fluorescence intensity of each experimental group to the wells without cells.

\section{Pyroptosis fluorescence assays}

Fluorescence experiments were performed to detect the degree of pyroptosis of HPMECs(13). The endothelial cells were seeded in a 24-well plate. The medium was changed with $1 \mathrm{ml}$ of fresh and complete culture on 
the next day, and then cultured for 1-2 days. When the cell confluency reached $80 \%-90 \%$, LPS $(1 \mu \mathrm{g} / \mathrm{ml})$ was added to the experimental group, and cultured the HPMECs for four hours. After the stimulation, cells in each group were washed twice with DPBS. Next, $96 \mu$ l of Annexin V-FITC binding solution, $1 \mu$ l of Annexin V-FITC (green fluorescence), and $12.5 \mu \mathrm{l}$ of PI (Propidium iodide, red fluorescence) were added to each well. After mixing, the samples were placed on ice, avoiding light for 10 minutes. Finally, an immunofluorescence microscope (Leica dm18, Germany) was used to observe the endothelial cell membrane staining. The Annexin V-FITC fluorescence intensity reflects the degree of PS exposure on the cell membrane surface. The PI fluorescence intensity represents the degree of cell membrane integrity damage.

\section{DNA pull-down and protein profiling}

In order to determine the possible transcription factors of PLSCR4, our study used the DNA pull-down and protein profiling techniques. T-75 flasks were used to cultivate HPMEC cells. These were divided into two groups named the control group and experimental group, and it was ensured that the number of cells in each group was larger than $2 \times 10^{7}$. The PLSCR4 promoter sequence probe was synthesized and labeled according to the NCBI gene library information, and two sets of cellular proteins were extracted for the DNA pull-down. After the probe of coupling biotin bonded with the magnetic beads that coupled streptomycin, the probe reacted with the sample, and the protein bound to the probe was obtained. After the one-dimensional or twodimensional electrophoresis, the gel strips and dots of the target protein were obtained, and the in-gel enzymatic hydrolysis method was optimized. If there was a clear difference between the experimental group ( exp group ) and control group ( ctrl group ), mass spectrometry was performed. In this study, LC-MS/MS technology was used to analyze and obtain relevant information, such as the charge and peak map of the protein fragmentation fragments, and the Proteinpilot database software was used to perform bioinformatics analysis on the obtained results. The mass spectrometry analysis result and the silver staining result was analyzed together to obtain the protein information on the target gel point, which might be the transcription factor of PLSCR4.

\section{Statistical analysis}

Each group of experiments was repeated for at least three times. The experimental data were expressed as mean \pm standard deviation. One-way ANOVA was used for comparisons between groups. All data were analyzed and processed using the SPSS 22.0 statistical software. $P<0.05$ indicated that the difference was statistically significant.

\section{Results}

\section{PLSCR4 is closely correlated to the pyroptosis and permeability of the HPMECs.}

In recent years, research on cell death methods has revealed a new type of programmed and inflammatory death-cell pyroptosis(14). The characteristic pathological changes of cell pyroptosis are accompanied by secretion of IL-1 $\beta$ and IL-18 release $(15,16)$, and damage to the integrity of the cell membrane. Hence, cell pyroptosis is also called cell inflammatory necrosis $(17,18)$. In order to verify the change of PLSCR4 expression in vascular endothelial cells and its correlation with the degree of pyroptosis and permeability 
change, HPMECs were divided into four groups, and seeded into six-well plates. Except that the first group, which was the control group, the remaining three groups were treated with LPS, PLSCR4 siRNA + LPS, and the transfection reagent, respectively. The PLSCR4 siRNA sequence (5' to 3') was

GGAAGUGGAAUGGUUUGUUTT. After four hours of LPS stimulation, western blot was performed to detect the changes in the expression of PLSCR4. At the same time, the supernatant of each group were collected, and ELISA was performed to detect the concentration of IL-1 $\beta$ and IL-18. The Tracer Flux Assay was used to detect the HPMEC permeability changes.

After four hours of LPS stimulation, the expression level of PLSCR4 in the LPS group significantly increased, when compared to the control group, which is consistent with the previous high-throughput sequencing results (Fig. 2A). After the PLSCR4 siRNA silencing, the expression level of PLSCR4 decreased, and the expression of PLSCR4 in the transfection reagent group indicated that the transfection reagent had almost no effect on the HPMECs. The ELISA results revealed that after LPS stimulation, the concentration of IL-1 $\beta$ and IL-18 in the cell culture medium significantly increased, compared to the control group. Furthermore, the increase in PLSCR4 siRNA + LPS group was more significant (Fig. 2B and 2C), suggesting that PLSCR4 may have the effect of reducing the release of IL-1 $\beta$ and IL-18 from HPMECs. The Tracer flux assay results revealed that the permeability of HPMECs in the LPS group significantly increased compared to the control group, and it increased more significantly in the group of PLSCR4 siRNA + LPS (Fig. 2D). These results above showed that with the decrease in PLSCR4 expression, the degree of inflammatory response of pyroptosis in HPMECs after the LPS stimulation, and the permeability of endothelium increased, suggesting that PLSCR4 may plays an important anti-inflammatory role in pyroptosis, and has a function of maintaining the endothelium permeability.

\section{PLSCR4 alleviates pyroptosis through PS, but has no effect on the proteins of the pyroptosis pathway.}

In order to verify the mechanism of PLSCR4 upon pyroptosis, HPMECs were grouped and seeded as above. The distribution of PS on the cell surface and the integrity of the cell membrane were observed using the fluorescence method. The binding characteristics of Annexin V-FITC (green fluorescence) with PS were used to detect the amount of PS on the cell membrane surface. PI (red fluorescence) was used to stain the nuclear DNA to reflect the integrity of the cell membrane. Western blot was performed to detect the effects of PLSCR4 on the proteins referring to pyroptosis.

Compared with the LPS group, the green fluorescence of Annexin V-FITC on the HPMECs surface of the PLSCR4 siRNA + LPS group decreased, while the red fluorescence of PI increased (Fig. 3A). However, there was no obvious change in the control group and transfection agent group, suggesting that when the expression of PLSCR4 was too low to transport PS outside of the cell membrane, the destruction of the cell membrane of HPMECs increased, and N-GSDMD might form more transmembrane pores, which was also consistent with the increase in the concentration of IL-1 $\beta$ and IL-18 in the cell culture medium. The western blot results revealed that when the expression level of PLSCR4 changed, the expression of pyroptosis-related proteins were not affected (Fig. 3B), suggesting that PLSCR4 could reduce the pyroptosis degree of HPMECs. Hence, it was presumed that PLSCR4 could reduce pyroptosis of HPMECs by transferring PS outside of the cell membrane, with no significant effect on the proteins of the pyroptosis pathway. 


\section{DNA pull-down and protein profiling technology to study the transcription factor of PLSCR4.}

In order to improve further understanding of upstream mechanism of PLSCR4, our study investigated the upstream transcription factor of PLSCR4 using DNA pull-down and protein profiling technology.

The silver staining results for the control group and experimental group revealed that there was a significant difference between these two groups at the $18.4 \mathrm{KDa}$ molecular weight (Fig. 4). In the protein profiling experiment, when the reliability was set to conf $\geq 95 \%$ and unique peptides $\geq 1$, the number of secondary spectra generated by the sample mass spectrometer were 6,830 and 2,765 respectively, and the number of resolved secondary spectra were 1,685 and 423 . Common contaminated proteins and matching peptides were filtered out. The overall situation of the proteins identified by the sample from control group are presented in Table 1, and the information of the proteins from experimental group are presented in Table 2 with a molecular weight of $18.4 \mathrm{KDa}$ was obtained (Table 2). 
Table 1

Information of top 20 proteins identified by the sample from control group.

\begin{tabular}{|lllllll|}
\hline Accession & Length & Mass(Da) & Unused & Coverage(\%) & Spectrum & $\begin{array}{l}\text { Uniq } \\
\text { spectrum }\end{array}$ \\
\hline sp|E9PAV3|NACAM_HUMAN & 2078 & 205419 & 4.01 & 1.396 & 6 & 6 \\
\hline sp|P48047|ATPO_HUMAN & 213 & 23277.1 & 6.01 & 16.429999 & 4 & 4 \\
\hline sp|P17655|CAN2_HUMAN & 700 & 79994.5 & 1.47 & 3.4290001 & 3 & 3 \\
\hline sp|P62910|RL32_HUMAN & 135 & 15859.7 & 2 & 9.6299998 & 1 & 1 \\
\hline sp|P17987|TCPA_HUMAN & 556 & 60342.9 & 2.86 & 3.7769999 & 2 & 2 \\
\hline sp|Q15366|PCBP2_HUMAN & 365 & 38579.7 & 2 & 3.836 & 2 & 2 \\
\hline sp|014776|TCRG1_HUMAN & 1098 & 123900.1 & 7.34 & 2.9139999 & 4 & 4 \\
\hline sp|Q9Y2W2|WBP11_HUMAN & 641 & 69997 & 2 & 1.716 & 1 & 1 \\
\hline sp|Q92522|H1X_HUMAN & 213 & 22487 & 2 & 4.6950001 & 1 & 1 \\
\hline sp|P42766|RL35_HUMAN & 123 & 14551.4 & 4 & 11.38 & 2 & 2 \\
\hline sp|P15924|DESP_HUMAN & 2871 & 331771.2 & 4.82 & 0.5225 & 2 & 2 \\
\hline sp|P04275|VWF_HUMAN & 2813 & 309261.8 & 6.24 & 1.209 & 4 & 4 \\
\hline sp|Q9BTM1|H2AJ_HUMAN & 129 & 14019.3 & 2 & 20.93 & 13 & 3 \\
\hline sp|P23528|COF1_HUMAN & 166 & 18502.3 & 3.15 & 13.249999 & 6 & 6 \\
\hline sp|Q00341|VIGLN_HUMAN & 1268 & 141454.5 & 4.09 & 2.366 & 3 & 3 \\
\hline sp|P27635|RL10_HUMAN & 214 & 24603.7 & 2.62 & 5.607 & 1 & 1 \\
\hline sp|P25398|RS12_HUMAN & 132 & 14514.8 & 2.15 & 6.8180002 & 2 & 2 \\
\hline sp|P10809|CH60_HUMAN & 573 & 61054.2 & 2 & 2.094 & 2 & 2 \\
\hline sp|P09661|RU2A_HUMAN & 255 & 28415.3 & 3.37 & 5.8820002 & 2 & 2 \\
\hline sp|Q12797|ASPH_HUMAN & 758 & 85862.1 & 3.16 & 2.2430001 & 3 & 3 \\
\hline Unused: protein identification score; Coverage: conf $\geq 95 \%$ protein & coverage; Spectrum: total spectrum \\
\hline amount; Uniq spectrum: unique spectrum amout. & & & & \\
\hline
\end{tabular}


Table 2

Information of proteins from experimental group treated with LPS.

\begin{tabular}{|c|c|c|c|c|c|c|}
\hline Accession & Length & Mass(Da) & Unused & Coverage(\%) & Spectrum & $\begin{array}{l}\text { Uniq } \\
\text { spectrum }\end{array}$ \\
\hline sp|Q7L7LO|H2A3_HUMAN & 130 & 14121.4 & 2.52 & 6.9229998 & 9 & 9 \\
\hline *sp|P62280|RS11_HUMAN & 158 & 18430.6 & 3.54 & 8.8610001 & 2 & 2 \\
\hline sp|Q00325|MPCP_HUMAN & 362 & 40094.5 & 6 & 8.0109999 & 3 & 3 \\
\hline sp|P07305|H10_HUMAN & 194 & 20862.8 & 1.66 & 4.6390001 & 1 & 1 \\
\hline sp|Q9Y324|FCF1_HUMAN & 198 & 23369.4 & 2 & 5.556 & 1 & 1 \\
\hline sp|P63261|ACTG_HUMAN & 375 & 41792.5 & 20.46 & 19.73 & 12 & 12 \\
\hline sp|P12236|ADT3_HUMAN & 298 & 32866 & 5.82 & 8.3889998 & 5 & 5 \\
\hline sp|P20073|ANXA7_HUMAN & 488 & 52738.9 & 2 & 2.2539999 & 1 & 1 \\
\hline sp|P0DPH8|TBA3D_HUMAN & 450 & 49959.1 & 8.47 & 7.9999998 & 6 & 6 \\
\hline sp|P06899|H2B1J_HUMAN & 126 & 13904.1 & 2.18 & 47.620001 & 16 & 1 \\
\hline sp|Q9HD33|RM47_HUMAN & 250 & 29450.1 & 1.33 & 3.2000002 & 1 & 1 \\
\hline sp|Q2VIR3|IF2GL_HUMAN & 472 & 51228.2 & 3.01 & 4.4489998 & 2 & 2 \\
\hline sp|P05121|PAl1_HUMAN & 402 & 45059.7 & 2.84 & 1.7410001 & 1 & 1 \\
\hline sp|060814|H2B1K_HUMAN & 126 & 13890 & 13.78 & 47.620001 & 17 & 2 \\
\hline sp|Q9H0S4|DDX47_HUMAN & 455 & 50646.1 & 2 & 1.7580001 & 1 & 1 \\
\hline sp|000422|SAP18_HUMAN & 153 & 17561 & 1.81 & 5.229 & 1 & 1 \\
\hline sp|P18206|VINC_HUMAN & 1134 & 123798.1 & 4.19 & 2.028 & 2 & 2 \\
\hline sp|P05362||CAM1_HUMAN & 532 & 57824.8 & 2 & 1.692 & 1 & 1 \\
\hline sp|P05388|RLA0_HUMAN & 317 & 34273.2 & 4.67 & 7.2559997 & 4 & 4 \\
\hline sp|Q96GQ7|DDX27_HUMAN & 796 & 89834.5 & 2 & 1.382 & 1 & 1 \\
\hline sp|P06703|S10A6_HUMAN & 90 & 10179.6 & 2 & 8.8890001 & 1 & 1 \\
\hline sp|Q14444|CAPR1_HUMAN & 709 & 78365.9 & 2.87 & 1.269 & 1 & 1 \\
\hline
\end{tabular}

P62280 may be involved as a transcription factor in the protective effect of PLSCR4 on HPMECs during ARDS. 
In order to verify the results for the DNA pull-down and protein profiling, and determine whether P62280 can be used as an upstream transcription factor to exert endothelial protection by regulating the expression of PLSCR4, HPMECs were divided into four groups, named the control group, LPS group, P62280 siRNA + LPS group, and transfection reagent group. The P62280 siRNA sequence ( $5^{\prime}$ to $\left.3^{\prime}\right)$ was

CCAAACGACTTGGACGATGTT, and the antibody of P62280 was purchased frome Abcam. Western blot was used to detect the expression of PLSCR4 and the pyroptosis-related proteins. ELISA was used to detect the degree of pyroptosis. Tracer Flux Assays were used to detect the permeability of endothelial cells during the pyroptosis. Finally, the fluorescence experiment was used to detect the degree of cell membrane integrity and the expression of PS on the cell surface after P62280 silencing.

After silencing P62280 by siRNA transfection, LPS continuously stimulated the HPMECs for four hours. It was found that the expression of PLSCR4 was consistent with the change in P62280. The changes in pyroptosis-related proteins, GSDMD, N-GSDM D, Caspase-1 and Caspaer-1 P20 were not affected by P62280 (Fig. 5A), suggesting that P62280 can regulate the expression level of PLSCR4, but has no significant effect on the classical pathway of pyroptosis.

When P62280 was silenced, the concentration of IL-1 $\beta$ and IL-18 in culture medium increased (Fig. 5B and $5 \mathrm{C}$ ), and the permeability was also significantly higher than that in the LPS group (Fig. 5D), suggesting that when the P62280 expression was reduced, the degree of pyroptosis increased, and the damage on barrier function of the membrane was aggravated. The fluorescence experiments revealed that when P62280 was silenced, the PS exposure on the surface of the pyroptosis cell membrane was reduced, compared to the simple LPS group, and the nuclear staining increased (Fig. 5E), suggesting that the integrity of the cell membrane was worse. Based on these results, it can be speculated that PLSCR4 played a protective role when HPMECs were stimulated by LPS, which mainly acted to reduce the degree of inflammation of pyroptosis to protect the integrity of the cell membrane, and maintain the barrier function of HPMECs. This protective effect of PLSCR4 may be conducted by transferring the PS on the inner surface of the cell membrane to the outside, and that reduced the binding of PS to the pyroptosis executive protein N-GSDMD, thereby inhibiting the formation of pyroptosis pores on the cell membrane.

\section{Discussion}

The acute lung injury or ARDS caused by sepsis appears as diffuse lung inflammation and pulmonary interstitial edema. The key step is the microvascular barrier dysfunction caused by the lung microvascular endothelial cell injury $(19,20)$. In the past, the mechanism of sepsis-induced pulmonary microvascular endothelial cell damage mainly focused on the endothelial cell death including necrosis, apoptosis, and cytoskeletal remodeling $(21,22)$. However, the exact mechanism remains inconclusive. Using high-throughput sequencing in the preliminary experiments of the research group, it was found that the PLSCR4 mRNA expression significantly increased after LPS stimulated HPMECs for four hours. However, the specific role and mechanism of PLSCR4 have not been reported yet.

PLSCRs are a class of single-pass transmembrane proteins that can transfer PS from the inside to the outside of the cell membrane in a $\mathrm{Ca}^{2+}$-dependent manner, increasing the exposure of PS. This process is 
involved in many pathophysiologies, such as cell phagocytosis, coagulation and inflammation, and plays an importang role(23-25). The research conducted by Wiedmer et al. confirmed that the expression levels of PLSCRs in humans and mice are different(6). Furthermore, there are more studies on PLSCR1-3 in the PLSCR family, while less research has been conducted on the specific effect of PLSCR4 in humans. Our study revealed that when LPS stimulated the HPMECs, the expression of PLSCR4, the concentration of the inflammatory factors IL-1 $\beta$ and IL-18, and the endothelial permeability all increased. After the silencing of PLSCR4, HPMEC inflammation and permeability more significantly increased, suggesting that PLSCR4 in endothelial cells have a protective effect. Then the next work was to study the mechanism of the protective effect. Studies have reported that the expression level of PLSCRs is consistent with the exposure level of PS (26-28).

PS is the most abundant negatively charged membrane phospholipid in the cell membrane. When cell is infected, PS is transported outside of the cell membrane by PLSCRs. The increased exposure of the outside of the PS membrane is correlated to various pathophysiological processes, such as apoptosis, pyroptosis and necroptosis. Among which, studies have revealed that PS is involved in the anchor of the pyroptosis executive protein GSDMD in the cell membrane. Hence, we inferred that PLSCR4 might play an important effect in the pyroptosis(29-31), especially in the early stage of infection(32). Pyroptosis is a new type of programmed cell death characterized with inflammation after necrosis and apoptosis(14). The main mechanism is that in the presence of noxious stimuli, caspase- 1 is activated into its active form caspase- 1 P20 (33).,Afterwards, caspase-1 P20 activates the proinflammatory IL-1 $\beta$ and IL-18 to their active forms. Secondly, caspase-1 P20 cleaves the GSDMD into an active N-terminal fragment N-GSDMD, which specifically binds to the inner PS(30) of the membrane and forms a homopolymer, a hydrophilic pores with a diameter of approximately $10-15 \mathrm{~nm}$ through the plasma membrane, destroying the integrity of the cell membrane. IL-1 $\beta$ and IL-18 are released to recruit other inflammatory cells, expanding the inflammatory response(34). Hence, pyroptosis is also called inflammatory necrosis of the cell, which can be detected for positive Annxin V-FITC staining. However, propidium iodide (PI) can stain the cell nucleus through the pores formed by N-GSDMD during pyroptosis, together with significant inflammatory response, which did not normally happen in the apoptosis. At the same time, unlike necrosis, the cell morphology usually remains normal in the early stage of pyroptosis(35), referring to the cellular defense response(36). Recent studies have reported that when stimulated by LPS, pulmonary vascular endothelial cells undergo extensive cell pyroptosis(37-39). Therefore, determining whether PLSCR4 exerts a protective effect on endothelial cells by regulating the distribution of PS has become the main concern of this study. In the results, it was found that when LPS stimulates HPMECs, with the increase in PLSCR4 expression, the Annexin V-FITC staining intensity of PS increased, and so did the nucleus staining with PI. When PLSCR4 was silenced, the PS exposure decreased, and the PI staining of the nucleus significantly increased, which is consistent with the change in inflammatory factors in the cell culture medium. This shows that when the expression of PLSCR4 decreases and PS could not be transported to the outside, the degree of pyroptosis of HPMECs increases. When the experiment used DNA pull-down and protein profiling experiments to further improve the mechanism of PLSCR4, it was found that PLSCR4 may be regulated by P62280 when stimulated by LPS.

\section{Conclusion}


In conclusion, our study is the first to reveal the protective effect of PLSCR4 and its mechanism. It was presumed that by transporting PS to the outside of the cell membrane, PLSCR4 could reduce the binding of the pyroptosis executive protein N-GSDMD to PS and the formation of pyroptosis pores, thereby reducing the degree of pyroptosis. The further discovery revealed that the expression of PLSCR4 mainly regulates by P62280. It is hoped that the experimental results of the present study can provide a new focus for studying endothelial cell damage, and new ideas for finding a solution for the clinical treatment of ARDS.

\section{Abbreviations}

PLSCR4: Phospholipid scramblase 4; ARDS: Acute respiratory distress syndrome; HPMEC: Pulmonary microvascular endothelial cell; LPS: lipopolysaccharide; PS: phospholipid; GSDMD: Gasdermin D; PI: permeability index.

\section{Declarations}

\section{Acknowledgements}

We would like to acknowledge the Medical Research Center of Shandong Provincial Qianfoshan Hospital and the Public Technical Service Platform of Shandong University for equipment support.

\section{Funding}

This work was supported by the National Natural Science Foundation of China (No.81770076), Academic promotion programme of Shandong First Medical University, Distinguished Taishan Scholars and Young Taishan Scholars.

\section{Availability of data and matetials}

The datasets used and analysed in the current study are not publicly available but are available from the corresponding author on reasonable request.

\section{Competinig Interest}

The authors declare that they have no competing interests.

\section{Authors' contributions}

$Y W$ designed the research; $\mathrm{XL}$ conducted the experiments, drafted the manuscript and prepared the figures and tables; DW and XZ analysed the data; $C G$ and FY edited and revised the manuscript; $Y W$ interpreted the results of the experiment and approve the final version of the manuscript. All authors read and approved the final manuscript.

\section{Consent for publication}

Not applicable. 


\section{Ethics approval and consent to participate}

Not applicable.

\section{References}

1. Akil A, Ziegeler S, Reichelt J, Rehers S, Abdalla O, Semik M, et al. Combined Use of CytoSorb and ECMO in Patients with Severe Pneumogenic Sepsis. The Thoracic and cardiovascular surgeon. 2020.

2. Auriemma CL, Zhuo H, Delucchi K, Deiss T, Liu T, Jauregui A, et al. Acute respiratory distress syndromeattributable mortality in critically ill patients with sepsis. Intensive care medicine. 2020.

3. Cheng KT, Xiong S, Ye Z, Hong Z, Di A, Tsang KM, et al. Caspase-11-mediated endothelial pyroptosis underlies endotoxemia-induced lung injury. J Clin Invest. 2017;127(11):4124-35.

4. Wang D, Gu C, Liu M, Liu G, Liu H, Wang Y. Analysis of Long Noncoding RNA Expression Profile in Human Pulmonary Microvascular Endothelial Cells Exposed to Lipopolysaccharide. Cell Physiol Biochem. 2019;52(4):653-67.

5. Wang D, Li Y, Gu C, Liu M, Wang Y. Identification of Key Pathways and Genes of Acute Respiratory Distress Syndrome Specific Neutrophil Phenotype. Biomed Res Int. 2019;2019:9528584.

6. Wiedmer T, Zhou Q, Kwoh DY, Sims PJ. Identification of three new members of the phospholipid scramblase gene family. Biochimica et biophysica acta. 2000;1467(1):244-53.

7. Bassé F, Stout JG, Sims PJ, Wiedmer T. Isolation of an erythrocyte membrane protein that mediates Ca2+-dependent transbilayer movement of phospholipid. The Journal of biological chemistry. 1996;271(29):17205-10.

8. Gaipl US, Beyer TD, Baumann I, Voll RE, Stach CM, Heyder P, et al. Exposure of anionic phospholipids serves as anti-inflammatory and immunosuppressive signal-implications for antiphospholipid syndrome and systemic lupus erythematosus. Immunobiology. 2003;207(1):73-81.

9. Mulvihill E, Sborgi L, Mari SA, Pfreundschuh M, Hiller S, Müller DJ. Mechanism of membrane pore formation by human gasdermin-D. Embo j. 2018;37(14).

10. Tsuchiya K, Nakajima S, Hosojima S, Thi Nguyen D, Hattori T, Manh Le T, et al. Caspase-1 initiates apoptosis in the absence of gasdermin D. Nat Commun. 2019;10(1):2091.

11. Chen J, Liu F, Lee SA, Chen S, Zhou X, Ye P, et al. Detection of IL-18 and IL-1beta protein and mRNA in human oral epithelial cells induced by Campylobacter concisus strains. Biochem Biophys Res Commun. 2019;518(1):44-9.

12. Bischoff I, Hornburger MC, Mayer BA, Beyerle A, Wegener J, Furst R. Pitfalls in assessing microvascular endothelial barrier function: impedance-based devices versus the classic macromolecular tracer assay. Sci Rep. 2016;6:23671.

13. He WT, Wan H, Hu L, Chen P, Wang X, Huang Z, et al. Gasdermin D is an executor of pyroptosis and required for interleukin-1beta secretion. Cell Res. 2015;25(12):1285-98.

14. Fink SL, Cookson BT. Apoptosis, pyroptosis, and necrosis: mechanistic description of dead and dying eukaryotic cells. Infect Immun. 2005;73(4):1907-16. 
15. He Y, Hara H, Nunez G. Mechanism and Regulation of NLRP3 Inflammasome Activation. Trends Biochem Sci. 2016;41(12):1012-21.

16. Lamkanfi M, Dixit Vishva M. Mechanisms and Functions of Inflammasomes. Cell. 2014;157(5):1013-22.

17. Bergsbaken T, Fink SL, Cookson BT. Pyroptosis: host cell death and inflammation. Nat Rev Microbiol. 2009;7(2):99-109.

18. Chen X, He WT, Hu L, Li J, Fang Y, Wang X, et al. Pyroptosis is driven by non-selective gasdermin-D pore and its morphology is different from MLKL channel-mediated necroptosis. Cell Res. 2016;26(9):1007-20.

19. Angus DC, van der Poll T. Severe sepsis and septic shock. The New England journal of medicine. 2013;369(9):840-51.

20. Bhattacharya J, Matthay MA. Regulation and repair of the alveolar-capillary barrier in acute lung injury. Annu Rev Physiol. 2013;75:593-615.

21. Wang L, Liu J, Xie W, Li G, Yao L, Zhang R, et al. Overexpression of MALAT1 Relates to Lung Injury through Sponging miR-425 and Promoting Cell Apoptosis during ARDS. Can Respir J. 2019;2019:1871394.

22. Chambers E, Rounds S, Lu Q. Pulmonary Endothelial Cell Apoptosis in Emphysema and Acute Lung Injury. Adv Anat Embryol Cell Biol. 2018;228:63-86.

23. Bevers EM, Comfurius P, Zwaal RF. Platelet procoagulant activity: physiological significance and mechanisms of exposure. Blood reviews. 1991;5(3):146-54.

24. Fadok VA, Voelker DR, Campbell PA, Cohen JJ, Bratton DL, Henson PM. Exposure of phosphatidylserine on the surface of apoptotic lymphocytes triggers specific recognition and removal by macrophages. Journal of immunology (Baltimore, Md : 1950). 1992;148(7):2207-16.

25. Verhoven B, Schlegel RA, Williamson P. Mechanisms of phosphatidylserine exposure, a phagocyte recognition signal, on apoptotic $T$ lymphocytes. The Journal of experimental medicine. 1995;182(5):1597-601.

26. Wolfs JL, Comfurius P, Rasmussen JT, Keuren JF, Lindhout T, Zwaal RF, et al. Activated scramblase and inhibited aminophospholipid translocase cause phosphatidylserine exposure in a distinct platelet fraction. Cellular and molecular life sciences : CMLS. 2005;62(13):1514-25.

27. Lang PA, Kempe DS, Akel A, Klarl BA, Eisele K, Podolski M, et al. Inhibition of erythrocyte "apoptosis" by catecholamines. Naunyn-Schmiedeberg's archives of pharmacology. 2005;372(3):228-35.

28. Zwaal RF, Comfurius P, Bevers EM. Scott syndrome, a bleeding disorder caused by defective scrambling of membrane phospholipids. Biochimica et biophysica acta. 2004;1636(2-3):119-28.

29. Ding J, Wang K, Liu W, She Y, Sun Q, Shi J, et al. Pore-forming activity and structural autoinhibition of the gasdermin family. Nature. 2016;535(7610):111-6.

30. Liu X, Zhang Z, Ruan J, Pan Y, Magupalli VG, Wu H, et al. Inflammasome-activated gasdermin D causes pyroptosis by forming membrane pores. Nature. 2016;535(7610):153-8.

31. Martinon F, Burns K, Tschopp J. The inflammasome: a molecular platform triggering activation of inflammatory caspases and processing of prolL-beta. Molecular cell. 2002;10(2):417-26. 
32. Galle JN, Hegemann JH. Exofacial phospholipids at the plasma membrane: ill-defined targets for early infection processes. Biological chemistry. 2019;400(10):1323-34.

33. Feria MG, Taborda NA, Hernandez JC, Rugeles MT. HIV replication is associated to inflammasomes activation, IL-1 $\beta$, IL-18 and caspase-1 expression in GALT and peripheral blood. PloS one. 2018;13(4):e0192845.

34. Schneider KS, Groß CJ, Dreier RF, Saller BS, Mishra R, Gorka O, et al. The Inflammasome Drives GSDMDIndependent Secondary Pyroptosis and IL-1 Release in the Absence of Caspase-1 Protease Activity. Cell reports. 2017;21(13):3846-59.

35. Vande Walle L, Lamkanfi M. Pyroptosis. Current biology : CB. 2016;26(13):R568-r72.

36. de Vasconcelos NM, Lamkanfi M. Recent Insights on Inflammasomes, Gasdermin Pores, and Pyroptosis. Cold Spring Harbor perspectives in biology. 2020;12(5).

37. He X, Qian Y, Li Z, Fan EK, Li Y, Wu L, et al. TLR4-Upregulated IL-1 $\beta$ and IL-1RI Promote Alveolar Macrophage Pyroptosis and Lung Inflammation through an Autocrine Mechanism. Sci Rep. 2016;6:31663.

38. Wu D, Pan P, Su X, Zhang L, Qin Q, Tan H, et al. Interferon Regulatory Factor-1 Mediates Alveolar Macrophage Pyroptosis During LPS-Induced Acute Lung Injury in Mice. Shock (Augusta, Ga). 2016;46(3):329-38.

39. Yang J, Zhao Y, Zhang P, Li Y, Yang Y, Yang Y, et al. Hemorrhagic shock primes for lung vascular endothelial cell pyroptosis: role in pulmonary inflammation following LPS. Cell death \& disease. 2016;7(9):e2363.

\section{Figures}




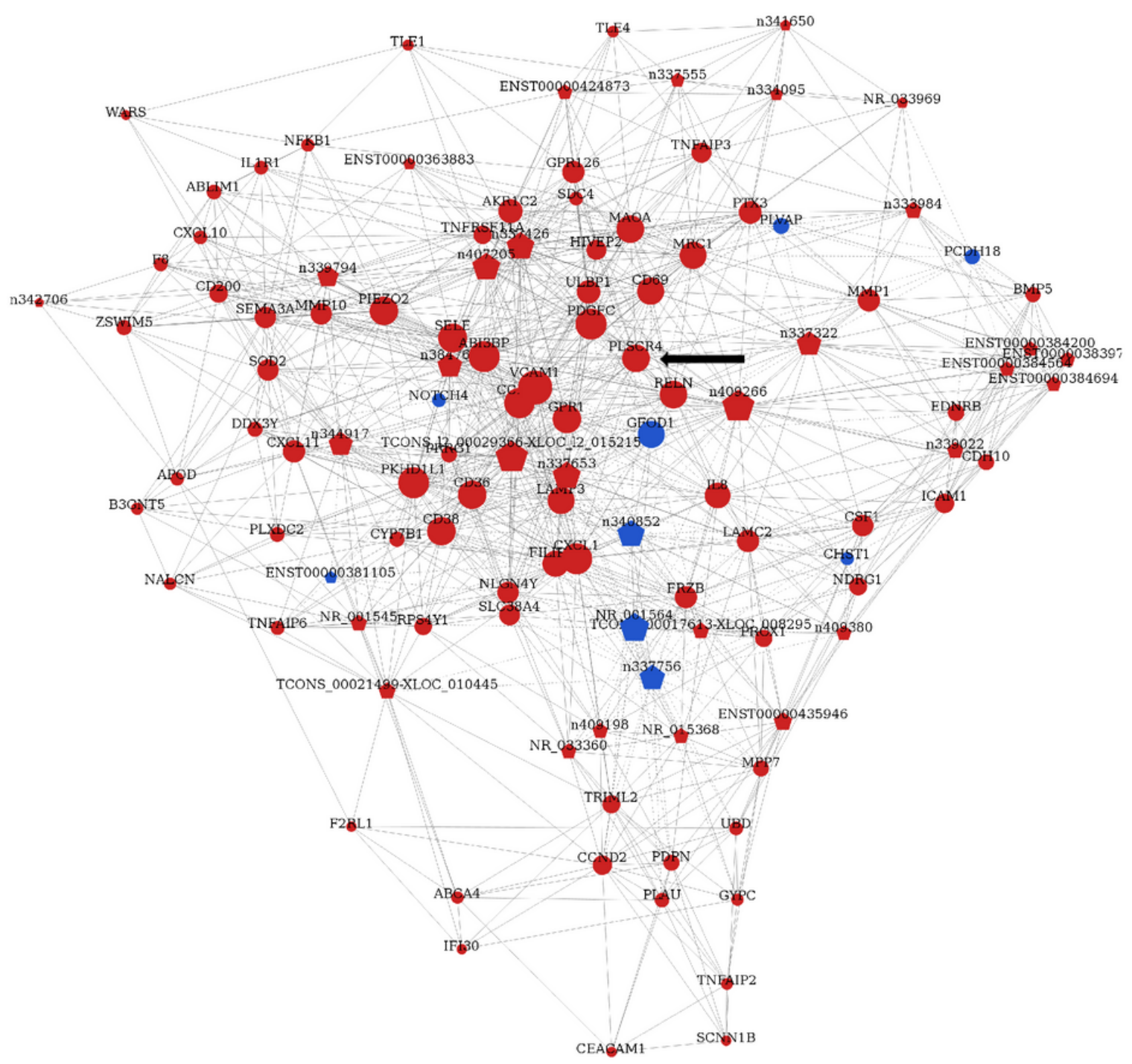

Figure 1

Coexpression network of differentially expressed mRNAs and interacting expressed IncRNAs in HPMECs exposed in LPS. The diamonds represent IncRNAs, and the circles represent mRNAs. Blue dots and red dots indicate downregulated and upregulated RNAs and the degree of upregulation and downregulation. The arrow indicates the expression of PLSCR4. 
A
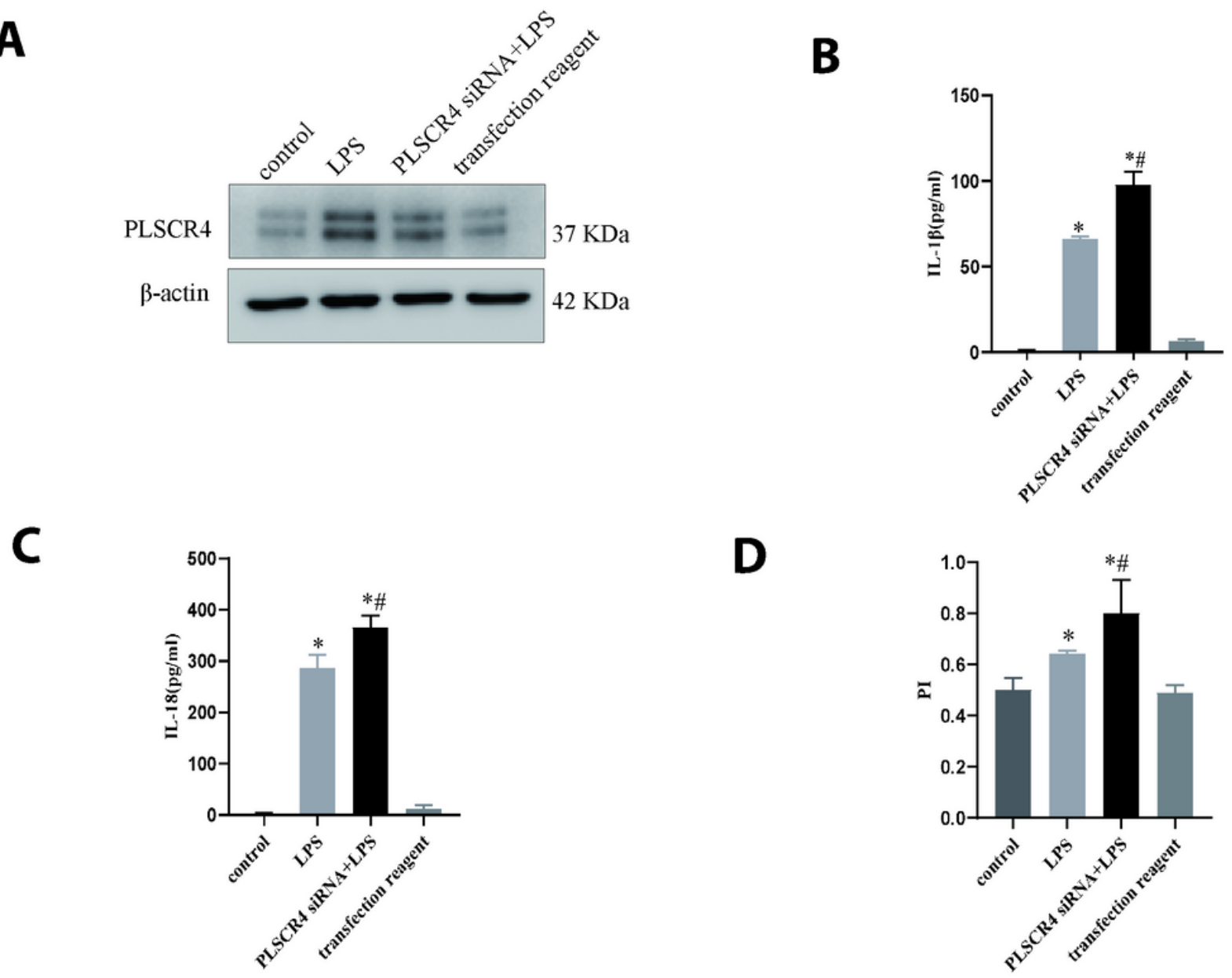

\section{Figure 2}

PLSCR4 was closely correlated to the pyroptosis and permeability changes of HPMEC. (A) The expression level of PLSCR4 significantly increased after LPS stimulation, and decreased after siRNA silencing. (B) and (C) After the PLSCR4 siRNA silencing, the concentration of IL-1 $\beta$ and IL-18 in the cell culture medium significantly increased. (D) After silencing the PLSCR4, the increase in HPMEC permeability during LPS stimulation was more significant than that in the LPS group. ${ }^{*} \mathrm{P}<.05$ vs the control group, \#P<.05 vs the LPS group. 


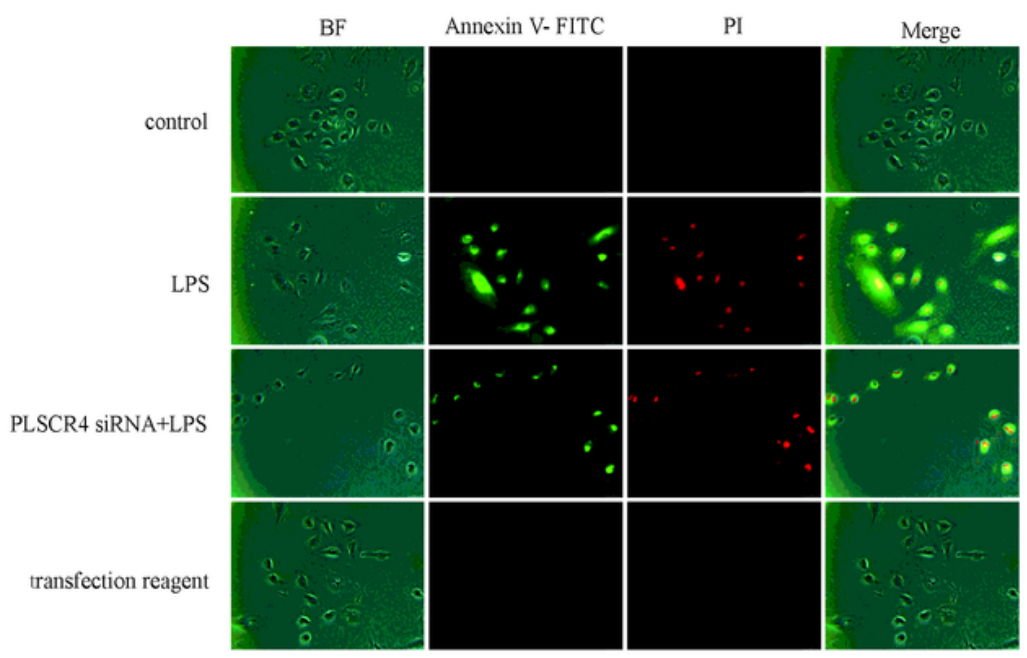

B

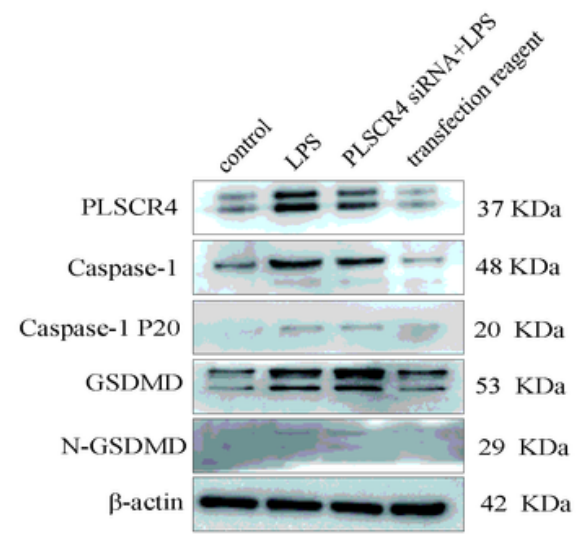

Figure 3

PLSCR4 affects cell pyrolysis through PS, but has no effect on the related protein activity of the pyrolysis pathway. (A) After inhibiting the expression of PLSCR4, there is less PS outside the cell membrane. Furthermore, the integrity of the cell membrane was destroyed, and the PI staining of the nucleus is enhanced. (B) The change in expression level of PLSCR4 had no significant effect on the pyrolysis-related proteins during cell pyrolysis. 


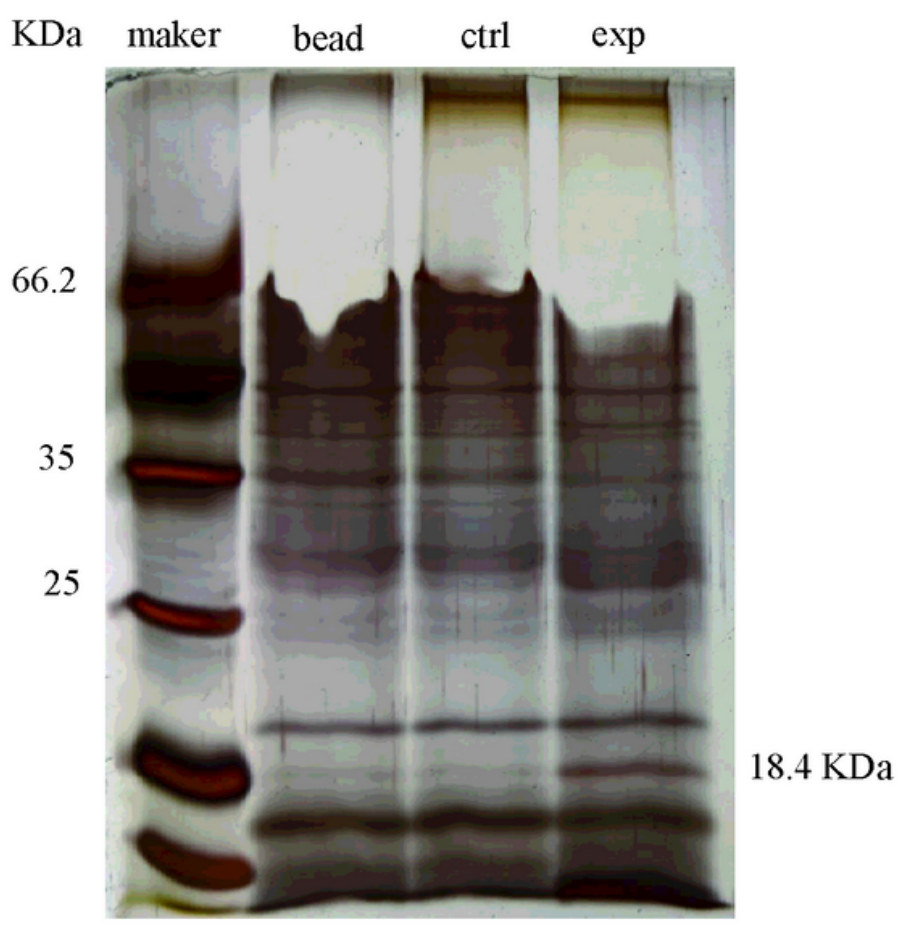

\section{Figure 4}

The SDS-PAGE silver staining results for the two groups of protein samples after DNA pull-down. The ctrl group is a blank control group. The exp group is the experimental group. The bead group is the blank magnetic bead control group without the DNA probe. The results revealed that at a molecular weight of 18.4 $\mathrm{KDa}$, the exp group was significantly different from the other groups. 
A

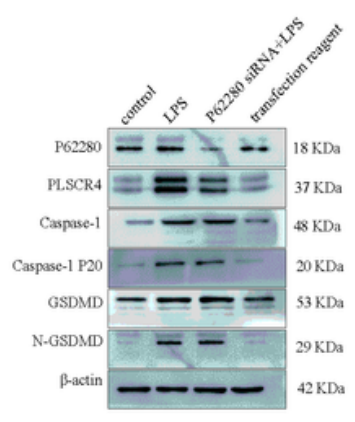

C

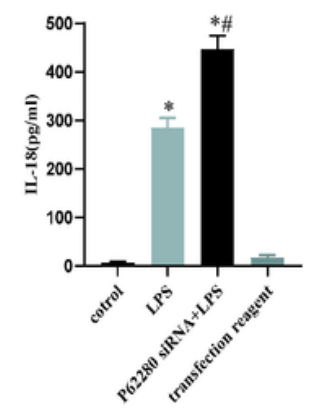

B

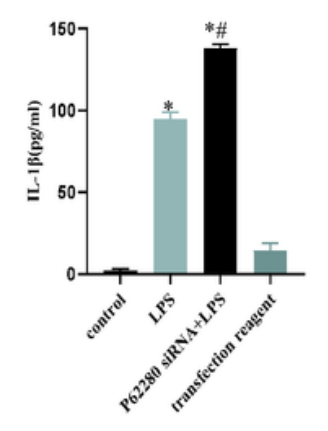

D

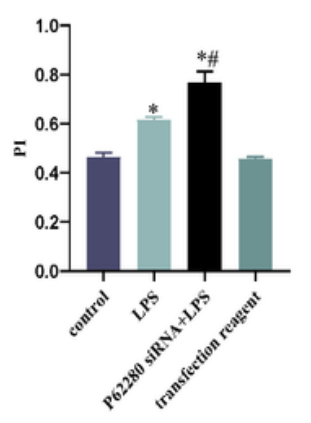

$\mathbf{E}$

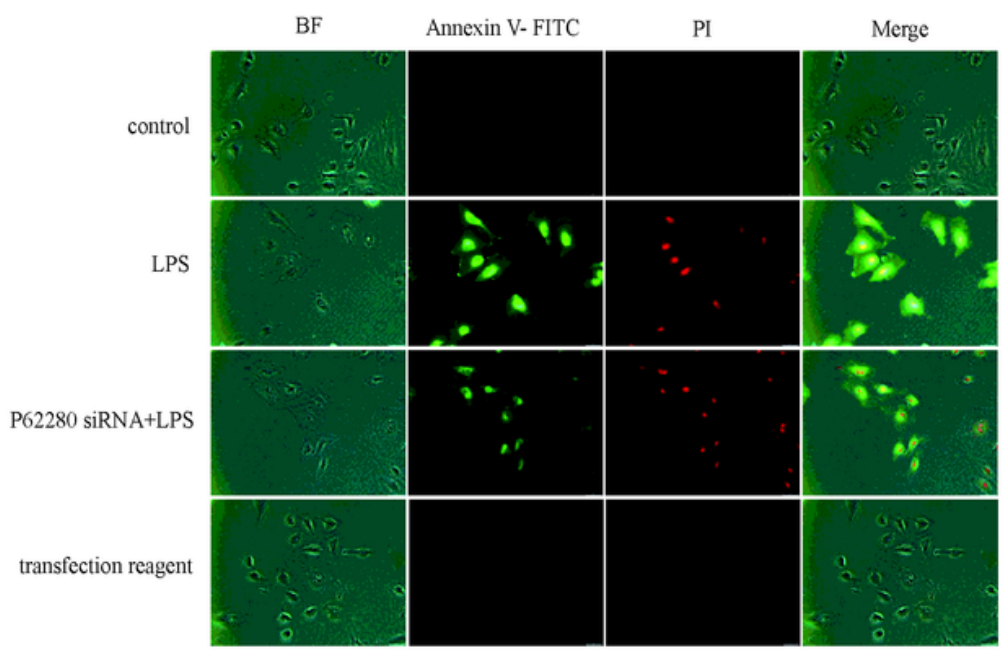

\section{Figure 5}

P62280 plays a role in inhibiting pyrolysis by regulating the expression level of PLSCR4. (A) After silencing P62280, the expression level of PLSCR4 decreased, but the related proteins of the pyrolysis pathway were not affected. (B, C and D) Compared to the LPS group, the concentration of IL-1 $\beta$ and IL-18 in the P62280 siRNA+LPS group was higher in the culture medium, and the endothelial permeability increased. (E) The Pyrodeath test revealed that the P62280 siRNA group had a higher degree of pyrolysis than the LPS group, 
and that the PS exposure on the outer surface of the cell membrane was reduced. ${ }^{*} \mathrm{P}<.05$ vs the control group, \#P<.05 vs the LPS group. 